\title{
Exciton dynamics in DNA oligomers studied by broadband deep-UV transient absorption spectroscopy
}

\author{
Benjamin Bauer, Malte Oppermann, and Majed Chergui* \\ Laboratoire de Spectroscopie Ultrarapide, Lausanne Centre for Ultrafast Science, Institut des \\ Sciences et Ingénierie Chimiques, School of Basic Sciences, Ecole Polytechnique Fédérale de \\ Lausanne, CH-1015 Lausanne, Switzerland
}

\begin{abstract}
We report broadband transient absorption measurements of adenine strands in the deep-UV $(250-370 \mathrm{~nm})$. By varying the strand length we resolve the interplay between inter-base stacking and exciton formation and dynamics in DNA oligomers.
\end{abstract}

Protection against photo damage of DNA takes place via excited state decay pathways to the ground state. Despite intense research activity over the past decade [1-3], the precise interplay between the electronic and nuclear structures of DNA model systems as well as the associated ultrafast, excited state population dynamics remain elusive. Excited state dynamics in DNA oligomers are complex due to the interactions between the chromophores. The primary interactions are due to $\pi-\pi$ stacking between consecutive nucleotides in a strand, leading to exciton states after excitation and the pairing interactions between neighbouring nucleotides in different strands. To get a better understanding of the excited state dynamics we isolate the impact of the stacking interaction by studying single strands. Due to the $\pi$ - $\pi$ stacking interaction the excited state is restricted to a single base, but may be delocalized over multiple coupled chromophores. This exciton shows different dynamics compared to the single base excited state, with conflicting interpretations regarding its localization length and temporal evolution within the strand [1,2].

We address this issue by applying femtosecond deep-UV transient absorption spectroscopy with exceptionally broadband probe pulses $(250-370 \mathrm{~nm})$, which allow us to resolve the full ground state bleach (GSB) and excited state absorption (ESA) dynamics in DNA single strands for the first time. By comparing the spectral signatures of the Deoxyadenosine monophosphate monomer, dimer $(\mathrm{dA})_{2}$ and 20 -mer $(\mathrm{dA})_{20}$ in physiological buffer solution, we are able to identify the dependence of the exciton dynamics on the oligomer structure.

The deep-UV setup which runs at $20 \mathrm{kHz}$ provides a broadband deep-UV continuum between 245-380 $\mathrm{nm}$. Details are given in references [4,5]. The instrument response

\footnotetext{
${ }^{*}$ Corresponding author: Majed.Chergui@epfl.ch
} 
function (IRF) is determined from the two photon absorption signal in a pure solvent [6], which here is $<180 \mathrm{fs}$ for all wavelengths.

In the case of the monomer sample, dAMP (Fig. 1a), we observe ground state bleach (GSB), stimulated emission (SE) and excited state absorption (ESA), resulting from a hot ground state, which experiences vibrational cooling on the picosecond time scale. The decays are mono exponential but the ESA and the SE signals have an energy dependent time constant. We observe a lifetime of $\tau_{\mathrm{GSB}}=0.8 \mathrm{ps}$ for GSB, lifetimes between $\tau_{\mathrm{ESA}}=0.3 \mathrm{ps}$ $(3.2 \mathrm{eV})$ and $\tau_{\mathrm{ESA}}=1.5 \mathrm{ps}(4.4 \mathrm{eV})$ for ESA and lifetimes between $\tau_{\mathrm{SE}}=60 \mathrm{fs}(3.2 \mathrm{eV})$ and $\tau_{\mathrm{SE}}=280 \mathrm{fs}(4.4 \mathrm{eV})$ for SE consistent with previous reports $[3,7]$.

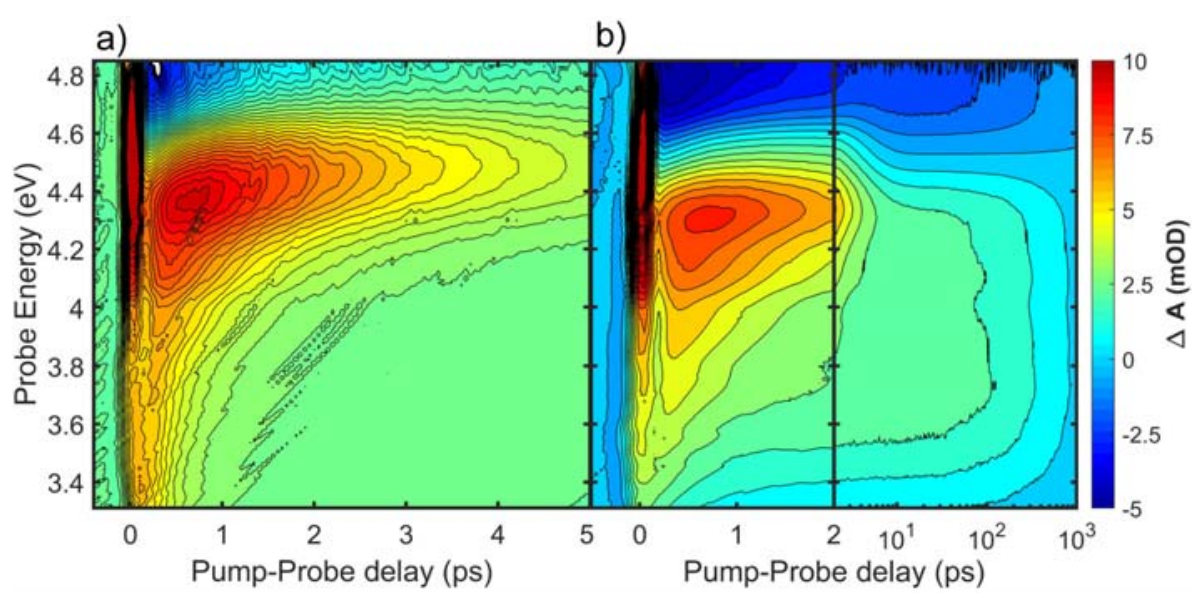

Fig. 1. Spectrally-resolved transient absorption signal of dAMP (a) and (dA) $)_{2}(b)$ in phosphate buffered aqueous solution at different pump-probe delays in a pseudo-colour contour representation. b) has a logarithmic time scale from $2 \mathrm{ps}$ on. All samples are pumped at $4.66 \mathrm{eV}$ and have an absorption of $80 \%$ at $4.77 \mathrm{eV}$.

Going to the deoxyadenosine dimer linked with a phosphate backbone $\left((\mathrm{dA})_{2}\right.$, Fig. 1b), the timescales as well as the spectral shape of the ESA change tremendously. The first few picoseconds are dominated by a monomer-like spectral change with lifetimes approximately 1.5 times longer compared to the monomer. Additionally, we observe a second slower decay process with an approximately $1 \mathrm{eV}$ (FWHM) broad plateau-like ESA spectral shape. The time constant of 385 ps is identical for both ESA and GSB. This decay and the associated broad ESA feature are due to the stacking interaction and are thus a signature of a two-base exciton. Indeed, suppressing stacking with a methanol solution makes this signature vanish.

In a 20-base DNA oligomer (dA) $)_{20}$ (Fig. 2b) the possibility of forming excitons which span over more than two bases is present $[1,2,8]$. The excitonic ESA signature is significantly broader and decays twice as fast in the 20-mer than in the dimer. We assign these spectral and temporal differences to the contributions of excitons spanning more than two bases. Additionally, the spectra for later times differ significantly, which suggests that the exciton states spanning more than two bases may not decay to a two base exciton as a gateway state to the ground state. 


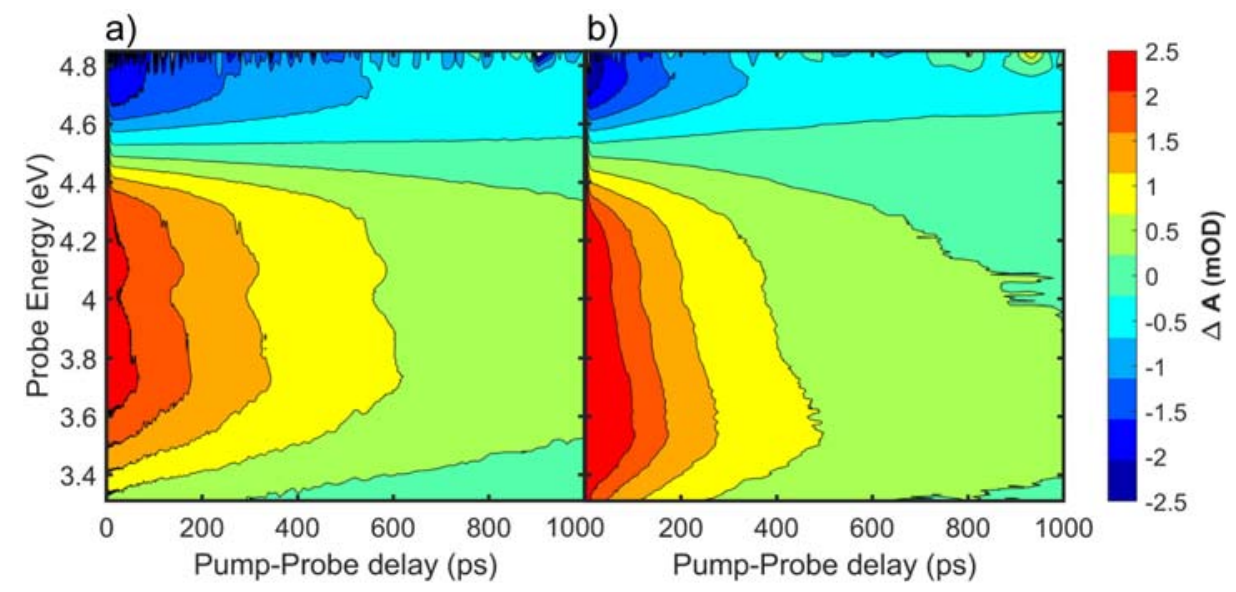

Fig. 2. Spectrally-resolved transient absorption signal of $(d A)_{2}$ (a) and $(d A)_{20}$ (b) in phosphate buffered aqueous solution at different pump-probe delays in a pseudo-colour contour representation. All samples are pumped at $4.66 \mathrm{eV}$ and have an absorption of $80 \%$ at $4.77 \mathrm{eV}$.

In the region where GSB and ESA absorption overlap there is a point where they cancel out and the overall transient absorption signal is zero. For $(\mathrm{dA})_{2}$, it appears around $4.55 \mathrm{eV}$ and is approximately independent of the time delay (Fig. 2a). In contrast in (dA) $)_{20}$ the zero-line shifts from $4.55 \mathrm{eV}$ at $50 \mathrm{ps}$ to $4.62 \mathrm{eV}$ at $1 \mathrm{~ns}$ (Fig. 2b). We therefore observe a spectral broadening of the ESA signal in the 20 -mer sample on the 100 ps time scale that is not present in the dimer sample. We assign this signature to conformational changes and/or exciton migration. The former being currently investigated by transient absorption anisotropy and the later by two-dimensional deep-UV (2DUV) spectroscopy.

This work was supported by the Swiss NSF through the NCCR-MUST and by a fellowship within the Postdoc.Program of the German Academic Exchange Service (DAAD).

\section{References}

1. I. Buchvarov Q. Wang, M. Raytchev, A. Trifonov, and T. Fiebig, PNAS 104, 47944797 (2007).

2. C. Su, C. Middleton and B. Kohler, J. Phys. Chem. B 116, 10266-10274 (2014).

3. D. Onidas, D. Markovitsi, S. Marguet, A. Sharonov, and T. Gustavsson, J. Phys. Chem. B. 106, 11367-11374 (2002).

4. G. Auböck, C. Consani, R. Monni, A. Cannizzo, F. v. Mourik and M. Chergui., Rev. Sci. Instrum. 83, 093105-6 (2012).

5. G. Auböck, C. Consani, F. v. Mourik and M. Chergui, Opt. Lett. 37, 2337-2339 (2012).

6. A. Reuther, A. Laubereau, D. N. Nikogosyan, Opt. Commun. 141,180-184 (1997).

7. C. T. Middleton, B. Cohen, and B. Kohler, J. Phys. Chem. A, 111, 10460-10467 (2007)

8. J. J. Nogueira, F. Plasser and L. González, Chem. Sci. 8, 5682-5691 (2017). 